\title{
Order-theoretic properties and separability of some sets of quasi-measures
}

\author{
Zbigniew Lipecki ${ }^{1}$
}

Received: 16 March 2017 / Revised: 5 July 2017 / Published online: 20 April 2018

(C) The Author(s) 2018

\begin{abstract}
Let $\mathfrak{M}$ and $\mathfrak{R}$ be algebras of subsets of a set $\Omega$ with $\mathfrak{M} \subset \mathfrak{R}$. Denote by $E(\mu)$ the set of all quasi-measure extensions of a given quasi-measure $\mu$ on $\mathfrak{M}$ to $\mathfrak{R}$. We present some results on the coincidence of the bands, in $\mathfrak{R}$, generated by $E(\mu)$ and extr $E(\mu)$. Moreover, we show that if $\mu$ is atomic, then $E(\mu)$ is contained in a principal band in $\Re$ if and only if it is separable. Another sufficient condition for the separability of $E(\mu)$ is also presented.
\end{abstract}

Keywords Linear lattice · Ideal · Band · Ideal dominated · Band dominated · Order unit - Weak order unit · Banach lattice $\cdot$ Order continuous norm · Separable $\cdot$ Convex set - Extreme point - Weakly compact - Additive set function · Quasi-measure . Atomic $\cdot$ Absolutely continuous $\cdot$ Extension

Mathematics Subject Classification 06F20 - 28A12 - 28A33 - 46A55 - 46B42

\section{Introduction}

By a quasi-measure we mean a positive additive function on an algebra of sets. Let $\mathfrak{M}$ and $\mathfrak{R}$ be algebras of subsets of a set $\Omega$ with $\mathfrak{M} \subset \mathfrak{R}$ and let $\mu$ be a quasi-measure on $\mathfrak{M}$. The 'sets' appearing in the title of the paper are the convex set $E(\mu)$ of all quasi-measure extensions of $\mu$ to $\Re$ and the set extr $E(\mu)$ of its extreme points. These

Communicated by P. De Lucia.

$凶$ Zbigniew Lipecki

lipecki@impan.pan.wroc.pl

1 Institute of Mathematics, Polish Academy of Sciences, Wrocław Branch, Kopernika 18, 51-617 Wrocław, Poland 
sets have been studied by the author in many earlier papers, including [8-11]. Of main concern in those papers have been their topological and linear-topological properties as subsets of the dual Banach lattice $b a(\Re)$. A systematic presentation of most of the results obtained is given in the memoir [12].

In contrast, $[13,14]$ and this paper are primarily concerned with order-theoretic properties of the sets $E(\mu)$. In [14] a property of subsets of a general linear lattice $X$ called ideal domination has been introduced and studied mainly in relation to $E(\mu)$ and extr $E(\mu)$. In Sect. 2 of this paper we introduce a property weaker than ideal domination, which we call band domination. (By definition, $V \subset X$ is band dominated if it is contained in a principal band in $X$.) We note that every separable subset of a Banach lattice is band dominated (Proposition 1(b)). We also show that band domination is strictly weaker than ideal domination in every infinite-dimensional Banach lattice (see Proposition 2).

In the Banach lattice $b a(\mathfrak{M})$, band domination is equivalent to domination in terms of $(\varepsilon-\delta)$ absolute continuity with respect to an element of $b a(\mathfrak{M})$, due to a classical result (see (PB) formulated between Remark 1 and Proposition 4 in Sect. 3). Therefore, every relatively weakly compact subset of $b a(\mathfrak{M})$ is band dominated, due to another classical result (see our comments after Lemma 5 in Sect. 4).

The main body of the paper consists of Sects. 5 and 6 . The former establishes, in the atomic case, a necessary and sufficient condition that the bands, in $b a(\Re)$, generated by $E(\mu)$ and extr $E(\mu)$ coincide (Theorem 1). As a consequence of an earlier result of the author [11], it is shown that those bands also coincide when $\mathfrak{R}$ is generated, as an algebra, by $\mathfrak{M}$ and a superatomic subalgebra of $\mathfrak{R}$, while $\mu$ is arbitrary (Theorem 2(a)).

In Sect. 6 four conditions equivalent to band domination of $E(\mu)$ in the atomic case are given, including the following one: extr $E(\mu)$ is separable (see Theorem 3 ). In the same case, assuming that $\mathfrak{R}$ is generated, as an algebra, by $\mathfrak{M}$ and another subalgebra $\mathfrak{N}$ of $\mathfrak{R}$ and $\mathfrak{M}, \mathfrak{N}$ and $\mu$ are related in a special way, we show that $E(\mu)$ is band dominated if and only if $\mathfrak{N}$ is countable and superatomic or $\mu=0$ (Corollary 2). Finally, we show that $E(\mu)$ is separable provided $\mu$ is separable (in the metric sense) and $\mathfrak{R}$ is generated, as an algebra, by $\mathfrak{M}$ and a countable family of pairwise disjoint subsets of $\Omega$ (Theorem 5).

The basic measure-theoretic terminology and notation we use are mostly standard. They are explained in Sect. 3, which also contains some auxiliary results of general character. Section 4 introduces some more notation and presents a number of auxiliary results on $E(\mu)$ and extr $E(\mu)$. There and in the remaining sections the material of [8-14] plays an essential role.

Some open questions are formulated throughout the paper; see the passage following Proposition 3 in Sect. 2, Remark 3 and the passage following Proposition 6, both in Sect. 3, and the passage following the proofs of Theorems $3^{\prime}$ and 4, both in Sect. 6.

It is a pleasure to dedicate this paper to Professor Hans Weber on his 70th birthday. I first met Hans in Wrocław during his visit to Poland in 1980, but we had started our mail contact a few years earlier. I have a pleasant memory of his hospitality in Radolfzell and Potenza and of our many meetings at conferences, mainly in Italy, Germany and Poland. I am grateful to him for his support during the state of emergency in Poland in the 1980s. The reading of Hans' papers has been instructive and inspiring for me. 
I have also reviewed some of them for MR and Zbl. I wish Hans many more interesting papers in the future as well as a long and active life in good health.

\section{Band domination in Banach lattices}

Let $X$ be a real linear lattice (= Riesz space in the terminology of [1]), with the order and lattice operations denoted by $\leqslant$ and $\wedge, \vee$, respectively. As usual, $|x|$ stands for the modulus or absolute value of $x \in X$ and $X_{+}$for the positive cone of $X$.

Let $V$ be a subset of $X$. We denote by $A_{V}$ [resp., $B_{V}$ ] the ideal [resp., band] in $X$ generated by $V$. Recall that a band is, by definition, an order closed ideal (see [1, p. 30]). An alternative name for a band is a normal linear sublattice (cf. [2, Definition 1.5.6]). The symbols $A_{\{x\}}$ and $B_{\{x\}}$, where $x \in X$, are abbreviated to $A_{x}$ and $B_{x}$, respectively. Ideals of the form $A_{x}$ and bands of the form $B_{x}$ are called principal.

We call $V$ ideal dominated [resp., band dominated] if $V \subset A_{x}$ [resp., $V \subset B_{x}$ ] for some $x \in X$. The former notion was introduced and studied in [14]. Note that $X$ is itself ideal dominated if and only if it has a (strong) order unit $e$, i.e., $e \in X_{+}$and for every $x \in X$ there exists $n \in \mathbb{N}$ with $|x| \leqslant n e$. Similarly, $X$ is itself band dominated if and only if it has a weak order unit $e$, i.e., $e \in X_{+}$and for every $x \in X_{+}$we have $x \wedge n e \uparrow x$ (see [1, p. 36]).

The following simple lemma will be instrumental in establishing the next proposition and Proposition 7(b),(c) in Sect. 4.

Lemma 1 Let $V_{1}, V_{2}, \ldots$ be subsets of a Banach lattice $X$. Then $\bigcup_{j=1}^{\infty} V_{j}$ is band dominated if (and only if) $V_{j}$ is band dominated for each $j$.

Proof Suppose $V_{j} \subset B_{x_{j}}$, where $x_{j} \in X$ and $j=1,2, \ldots$ By [14, Proposition 1], there exists $x \in X$ such that $x_{j} \in I_{x}$ for each $j$. Hence $\bigcup_{j=1}^{\infty} V_{j} \subset B_{x}$.

Part (b) of the following proposition will be applied several times below. Part (a) thereof is a direct consequence of the assertion that bands in a Banach lattice are closed (see [1, Theorem 11.2(3)]), and so weakly closed. We shall tacitly use this assertion also on some other occasions.

Proposition 1 Let $V$ be a subset of a Banach lattice $X$.

(a) If $V$ is band dominated, then so is its weak closure.

(b) If $V$ is separable, then it is band dominated.

Proof (b): Apply (a) and Lemma 1.

An analogue of Proposition 1(a) for subsets of a dual Banach lattice and the weak* topology fails; see Example 1 in Sect. 3.

There are many standard examples of Banach lattices without order unit that still have a weak order unit. A general result to this effect is a consequence of Proposition 1(b) and the following one.

Proposition 2 Every infinite-dimensional Banach lattice X contains a separable closed sublattice $X_{0}$ without order unit. 
Proof Without loss of generality, we assume that $X$ is separable (see [1, Exercise 9 of Section 12]). We also assume that $X$ has an order unit. Then it is isomorphic as a Banach lattice to a $\mathcal{C}(Z)$-space, where $Z$ is an (infinite) compact space, by a combination of classical results (see [1, Theorems 12.20 and 12.28 and Corollary 12.4]). Let $z_{0}$ be an accumulation point of $Z$, and set

$$
X_{0}=\left\{f \in \mathcal{C}(Z): f\left(z_{0}\right)=0\right\}
$$

It is easy to see that $X_{0}$ is as desired.

We note that a special class of sets for which ideal and band dominations coincide is described in Proposition 10 in Sect. 6.

By an application of the Krein-Milman theorem, we get the following result.

Proposition 3 Let $W$ be a weakly compact convex subset of a Banach lattice X. Then

$$
B_{W}=B_{\text {extr } W}
$$

Consequently, if extr $W$ is band dominated, then so is $W$.

The first assertion of Proposition 3 fails for weak* compact convex subsets of a dual Banach lattice; see Remark 2 in Sect. 3 and Remark 4 in Sect. 5. The author does not know whether the same can be said about the second assertion of Proposition 3.

\section{Further notation and measure-theoretic preliminaries}

Let $A$ be a Boolean algebra. The set of nonzero $\{0,1\}$-valued additive functions on $A$ is denoted by $u l t(A)$. Equipped with an appropriate topology, it can be identified with the Stone space of $A$. Recall that $A$ is said to be superatomic if every subalgebra of $A$ is atomic or, equivalently, every homomorphic image of $A$ is atomic (see [7, Proposition 17.5]; cf. also [2, Definition 5.3.4]). This notion often appears below, especially in Sects. 5 and 6.

The algebra of open-and-closed subsets of a topological space $Z$ is denoted by $\mathrm{CO}(Z)$.

For a set $\Omega$ we denote by $|\Omega|$ the cardinality of $\Omega$.

Throughout the rest of the paper, $\Omega$ stands for a nonempty set and $\mathfrak{M}$ for an algebra of subsets of $\Omega$.

Given a family $\mathfrak{E}$ of subsets of $\Omega$, we denote by $\mathfrak{E}_{b}$ the algebra of subsets of $\Omega$ generated by $\mathfrak{E}$.

We denote by $b a(\mathfrak{M})$ the Banach lattice of all real-valued bounded additive functions on $\mathfrak{M}$ (see [2, Section 2.2]). By definition, $\|\varphi\|=|\varphi|(\Omega)$ for $\varphi \in b a(\mathfrak{M})$. In addition to the strong topology, $b a(\mathfrak{M})$ is equipped with its weak and weak* topologies; see [2, Section 4.7] or [3, Section IV.5] for the canonical Banach-lattice predual of $b a(\mathfrak{M})$. 
We set

$$
\begin{aligned}
p a(\mathfrak{M}) & =\left\{\mu \in b a_{+}(\mathfrak{M}): \mu(\Omega)=1\right\} \\
u l t(\mathfrak{M}) & =\{\mu \in p a(\mathfrak{M}): \mu \text { is two-valued }\}
\end{aligned}
$$

With $\mu \in b a_{+}(\mathfrak{M})$ we associate the pseudometric $d_{\mu}$ on $\mathfrak{M}$ defined by the formula

$$
d_{\mu}\left(M_{1}, M_{2}\right)=\mu\left(M_{1} \triangle M_{2}\right), \quad M_{1}, M_{2} \in \mathfrak{M}
$$

We say that $\mu$ is separable if the corresponding pseudometric space is separable.

Let $\mu \in b a_{+}(\mathfrak{M})$. Adapting a general linear-lattice-theoretical terminology (see $[1, \mathrm{p} .13])$, we say that $\nu \in b a_{+}(\mathfrak{M})$ is a component of $\mu$ if

$$
v \wedge(\mu-v)=0
$$

We denote by $\mathcal{U}_{\mu}$ the set of all components of $\mu$ which take at most two values. As easily seen (cf. [2, Proposition 5.2.2]), for different $v_{1}, v_{2} \in \mathcal{U}_{\mu}$ we have $v_{1} \wedge v_{2}=0$. Therefore, $\mathcal{U}_{\mu}$ is countable. We say that $\mu$ is (purely) atomic if $\mu=\sum_{\nu \in \mathcal{U}_{\mu}} \nu$.

Remark 1 Suppose $\mu \in b a_{+}(\mathfrak{M})$ is atomic and $\mathcal{U}_{\mu}$ is finite. Then there exists an $\mathfrak{M}$-partition $\left\{M_{\nu}: v \in \mathcal{U}_{\mu}\right\}$ of $\Omega$ such that

$$
\mu\left(M \cap M_{\nu}\right)=v(M) \text { for all } M \in \mathfrak{M} \text { and } \nu \in \mathcal{U}_{\mu}
$$

It follows that the finite algebra

$$
\left\{M_{v}: v \in \mathcal{U}_{\mu}\right\}_{b}
$$

is $d_{\mu}$-dense in $\mathfrak{M}$, and so $\mu$ is separable. Consequently, every atomic $\mu \in b a_{+}(\mathfrak{M})$ is separable.

For $\varphi, \psi \in b a(\mathfrak{M})$ we write $\psi \ll \varphi$ if $\psi$ is absolutely continuous with respect to $\varphi$, i.e., the familiar $(\varepsilon-\delta)$ condition holds for $|\varphi|$ and $|\psi|$ (see [2, Definition 6.1.1]).

The following result will be frequently applied below.

(PB) For every $\varphi \in$ ba $(\mathfrak{M})$ we have $B_{\varphi}=\{\psi \in$ ba $(\mathfrak{M}): \psi \ll \varphi\}$.

This is a consequence of Theorems 1.5.12 and 6.2.2 of [2].

The next two propositions are more or less known. They will be used in Sect. 4 to establish Lemmas 4 and 5, respectively.

Proposition 4 The following two conditions are equivalent:

(i) $b a(\mathfrak{M})=B_{\text {ult }(\mathfrak{M}) \text {; }}$

(ii) $\mathfrak{M}$ is superatomic.

This is an immediate consequence of $[2$, Theorem 5.3.6, (iii) $\Rightarrow$ (i) and (i) $\Rightarrow$ (v)]. 
Remark 2 Condition (i) of Proposition 4 can be reformulated as follows:

$$
B_{\text {pa }(\mathfrak{M})}=B_{\text {ult }(\mathfrak{M})}
$$

Now, $p a(\mathfrak{M})$ is a weak* compact convex subset of $b a(\mathfrak{M})$ and extr $p a(\mathfrak{M})=\operatorname{ult}(\mathfrak{M})$, by well-known results (see, e.g., [12, p. 14]). Thus, Proposition 4 shows that Proposition 3 does not extend to weak* compact convex subsets of dual Banach lattices. Another example to the same effect is mentioned in Remark 4 in Sect. 5.

Proposition 5 The following six conditions are equivalent:

(i) ba(M) has a weak order unit;

(ii) ult $(\mathfrak{M})$ is band dominated;

(iii) ba $(\mathfrak{M})$ is separable;

(iv) $\operatorname{ult}(\mathfrak{M})$ is separable;

(v) $\mathfrak{M}$ is countable and superatomic;

(vi) ult $(\mathfrak{M})$ is countable.

Proof Clearly, (i) implies (ii) and (vi) implies (iv). By Proposition 1(b), (iii) implies (i) and (iv) implies (ii). To complete the argument, we shall establish the implications (ii) $\Rightarrow$ (vi) $\Rightarrow$ (v) $\Rightarrow$ (iii).

Suppose (ii) holds, and let $\tau \in b a_{+}(\mathfrak{M})$ be such that $\pi \ll \tau$ for every $\pi \in$ ult $(\mathfrak{M})$ (see (PB)). According to the Lebesgue decomposition theorem [2, Theorem 6.2.4], there exists $\tau_{\pi} \in b a_{+}(\mathfrak{M})$ with

$$
\tau_{\pi} \ll \pi \text { and }\left(\tau-\tau_{\pi}\right) \wedge \pi=0, \quad \text { where } \pi \in \operatorname{ult}(\mathfrak{M}) \text {. }
$$

We have $\tau_{\pi} \neq 0$ and $\tau_{\pi} \wedge \tau_{\pi^{\prime}}=0$ whenever $\pi, \pi^{\prime} \in u l t(\mathfrak{M})$ and $\pi \neq \pi^{\prime}$. Therefore, for each finite subset $\mathcal{F}$ of $u$ lt $(\mathfrak{M})$ we have $\tau \geqslant \sum_{\pi \in \mathcal{F}} \tau_{\pi}$. This implies (vi).

Suppose (vi) holds. Then $\mathfrak{M}$ is countable, by [7, Theorem 5.31]. Moreover, $\mathfrak{M}$ does not contain a tree, because otherwise we would have $|\operatorname{ult}(\mathfrak{M})| \geqslant 2^{\aleph_{0}}$, by a standard argument. Therefore, $\mathfrak{M}$ is superatomic (see [2, Theorem 5.3.6, (ii) $\Rightarrow$ (i)]), and so (v) holds.

Suppose (v) holds. By [7, Proposition 17.10], (vi) then holds, too. Therefore, (iii) follows, by [2, Theorem 5.3.6, (i) $\Rightarrow(\mathrm{v})]$.

Remark 3 The equivalence of conditions (iii) and (iv) in Proposition 5 suggests the question of whether $b a(\mathfrak{M})$ and $u l t(\mathfrak{M})$ always have the same density character. Under Martin's axiom, the answer is negative due to an example of Fremlin and Plebanek [4, Theorem 3A]. They construct a (zero-dimensional) compact space $Z$ of cardinality $\mathfrak{c}$ which carries $2^{\mathfrak{c}}$ Radon measures. Consequently, $|p a(\operatorname{CO}(Z))|=2^{\mathfrak{c}}$. This example also sheds some light on a result of Haydon [5] and Kadec and Fonf [6] used in the proof of Theorem 3' in Sect. 6 (cf. Remark 2).

The following example was announced after the proof of Proposition 1.

Example 1 Let $V$ be the linear span of the standard basis of the Banach lattice $l_{1}$. Then $V$ is ideal dominated, by [14, Proposition 1]. Moreover, according to a classical 
result (see [1, Theorem 10.5]), $V$ is weak ${ }^{*}$ dense in $l_{1}^{* *}$. Now, $l_{1}^{* *}$ can be identified as a Banach lattice with $b a\left(2^{\mathbb{N}}\right)$; cf. [2, Section 4.7] or [3, Section IV.5] for details. Finally, in view of [16, Proposition 0.5.9] or Proposition 5, (i) $\Rightarrow(v)$, above, $b a\left(2^{\mathbb{N}}\right)$ is not band dominated.

As already mentioned in the passage introducing Proposition 1, bands in Banach lattices are weakly closed. In this connection it is worth-while recalling that the following two conditions are equivalent for a Banach lattice $X$ :

(i) the norm of $X$ is order continuous, i.e., for every net $\left\{x_{\alpha}\right\}$ in $X$ with $x_{\alpha} \downarrow 0$ we have $\left\|x_{\alpha}\right\| \rightarrow 0$;

(ii) every band in $X^{*}$ is weak* closed;

see [1, Theorem 1.10] or [16, Theorem 1.19]. Combined with another result (see [16, Theorem 1.4]), this yields the following corollary: $b a(\mathfrak{M})$ contains a band which is not weak* closed provided $\mathfrak{M}$ is infinite. We shall strengthen this corollary as follows:

Proposition 6 If $\mathfrak{M}$ is infinite, then ba(M) contains a principal band which is not weak* closed.

Proof Due to the Stone representation theorem, we may assume that $\Omega$ is a (zerodimensional) compact space and $\mathfrak{M}=\mathrm{CO}(\Omega)$. By assumption, $\Omega$ is infinite. Fix $\omega_{0}$, $\omega_{n} \in \Omega, n=1,2, \ldots$ such that $\omega_{n} \neq \omega_{0}$ for each $n$ and $\omega_{0}$ is an accumulation point of the set $\left\{\omega_{n}: n=1,2, \ldots\right\}$. Denote by $\delta_{\omega_{0}}, \delta_{\omega_{n}}$ the corresponding Dirac quasi-measures on $\mathfrak{M}$. Then $\delta_{\omega_{0}}$ is a weak* accumulation point of the set $\left\{\delta_{\omega_{n}}: n=\right.$ $1,2, \ldots\}$, but

$$
\delta_{\omega_{0}} \notin B \sum_{n=1}^{\infty} 2^{-n} \delta_{\omega_{n}} .
$$

Thus, the assertion is established.

It is not clear to the author to what dual Banach lattices can Proposition 6 be generalized.

In the proof of Theorem 5 in Sect. 6 we shall apply the following two lemmas. The first of them is well known in the case where $\mathfrak{M}$ is a $\sigma$-algebra and $\mu$ is $\sigma$-additive. In fact, it can be reduced to that case, but we prefer to establish it in a more direct way.

Lemma 2 For $\mu \in b a_{+}(\mathfrak{M})$ the following two conditions are equivalent:

(i) $\mu$ is separable;

(ii) $B_{\mu}$ is separable.

Proof (cf. [11, proof of Lemma 1]). For every $N \in \mathfrak{M}$ define $\mu_{N} \in b a_{+}(\mathfrak{M})$ by

$$
\mu_{N}(M)=\mu(M \cap N), \quad M \in \mathfrak{M} .
$$

Clearly $\mu_{N} \in B_{\mu}$. Given $N_{1}, N_{2} \in \mathfrak{M}$, we have

$$
\mu_{N_{1}}-\mu_{N_{2}}=\mu_{N_{1} \backslash N_{2}}-\mu_{N_{2} \backslash N_{1}},
$$


and so

$$
\left\|\mu_{N_{1}}-\mu_{N_{2}}\right\|=\mu\left(N_{1} \triangle N_{2}\right) .
$$

Therefore, (ii) implies (i). On the other hand, $B_{\mu}$ coincides with the closed linear span of the set $\left\{\mu_{N}: N \in \mathfrak{M}\right\}$, by (PB) and [2, Theorem 6.3.4, (i) $\Rightarrow$ (iii)]. It follows that (i) implies (ii).

Lemma 3 Let $\mathfrak{M}=\left(\mathfrak{M}_{1} \cup \mathfrak{M}_{2}\right)_{b}$, where $\mathfrak{M}_{1}$ and $\mathfrak{M}_{2}$ are subalgebras of $\mathfrak{M}$, and $\mu \in b a_{+}(\mathfrak{M})$. If $\mu \mid \mathfrak{M}_{1}$ and $\mu \mid \mathfrak{M}_{2}$ are separable, then so is $\mu$.

Proof Let $\mathfrak{L}_{i}$ be a countable subfamily of $\mathfrak{M}_{i}$ which is $d_{\mu}$-dense in $\mathfrak{M}_{i}, i=1,2$. Set

$$
\mathfrak{L}=\left\{L_{1} \cap L_{2}: L_{1} \in \mathfrak{L}_{1} \text { and } L_{2} \in \mathfrak{L}_{2}\right\}
$$

Since

$$
\left(M_{1} \cap M_{2}\right) \triangle\left(L_{1} \cap L_{2}\right) \subset\left(M_{1} \Delta L_{1}\right) \cup\left(M_{2} \Delta L_{2}\right),
$$

the $d_{\mu}$-closure of $\mathfrak{L}$ contains the family

$$
\left\{M_{1} \cap M_{2}: M_{1} \in \mathfrak{M}_{1} \text { and } M_{2} \in \mathfrak{M}_{2}\right\}
$$

It follows that $\mathfrak{L}_{b}$ is $d_{\mu}$-dense in $\mathfrak{M}$.

\section{Auxiliary results on $E(\mu)$ and extr $E(\mu)$}

Throughout the rest of the paper, $\mathfrak{R}$ stands for an algebra of subsets of $\Omega$ with $\mathfrak{M} \subset \mathfrak{R}$.

We shall need the following notation (see [12, p. 14]). Given $\mu \in b a_{+}(\mathfrak{M})$, we set

$$
\mathfrak{J}_{\mu}=\{R \in \mathfrak{R}: \text { there exists } M \in \mathfrak{M} \text { with } R \subset M \text { and } \mu(M)=0\} .
$$

Clearly, $\mathfrak{J}_{\mu}$ is an ideal in $\mathfrak{R}$.

We also set

$$
E(\mu)=\left\{\varrho \in b a_{+}(\mathfrak{R}): \varrho \mid \mathfrak{M}=\mu\right\}
$$

It is a classical result that $E(\mu)$ is always nonempty (see [2, Chapter 3]). Moreover, it is, clearly, convex. In addition, it is weak* compact (see [12, Proposition 4.4(a)] or [8, Proposition 1(a)]).

The following proposition will be used in the proofs of the next one as well as of Theorem 3 and Proposition 10 in Sect. 6. 
Proposition 7 Suppose $\mu, \mu_{j} \in b a_{+}(\mathfrak{M})$ are such that $\sum_{j=1}^{\infty} \mu_{j}=\mu$ and $\mu_{j} \wedge \mu_{j^{\prime}}=$ 0 whenever $j \neq j^{\prime}$.

(a) $E\left(\mu_{j}\right) \subset A_{E(\mu)}$ and extr $E\left(\mu_{j}\right) \subset A_{\operatorname{extr} E(\mu)}$ for each $j$.

(b) $B_{E(\mu)}=B_{\cup_{j=1}^{\infty} E\left(\mu_{j}\right)}$; consequently, $E(\mu)$ is band dominated if and only if $E\left(\mu_{j}\right)$ is band dominated for each $j$.

(c) $B_{\mathrm{extr} E(\mu)}=B_{\cup_{j=1}^{\infty} \operatorname{extr} E\left(\mu_{j}\right)}$; consequently, extr $E(\mu)$ is band dominated if and only if extr $E\left(\mu_{j}\right)$ is band dominated for each $j$.

Proof (a): By [14, p. 202, formulas (1) and (2)] applied to $\mu_{j}$ and $\mu-\mu_{j}$, we get

$$
E(\mu)=E\left(\mu_{j}\right)+E\left(\mu-\mu_{j}\right) \text { and } \operatorname{extr} E(\mu)=\operatorname{extr} E\left(\mu_{j}\right)+\operatorname{extr} E\left(\mu-\mu_{j}\right) .
$$

The assertion follows.

(b): The inclusion " $\supset$ " is a consequence of the first part of (a). To show the converse inclusion, fix $\varrho \in E(\mu)$, and take $\varrho_{j} \in E\left(\mu_{j}\right), j=1,2, \ldots$, such that $\sum_{j=1}^{\infty} \varrho_{j}=\varrho$ (see [12, Theorem 6.1(a)] or [9, Theorem 1(a)]). We have

$$
\sum_{j=1}^{k} \varrho_{j} \uparrow \varrho, \quad \text { and so } \varrho \in B_{\bigcup_{j=1}^{\infty} E\left(\mu_{j}\right)} .
$$

The second part of (b) now follows from Lemma 1.

The proof of (c) is similar to that of (b). The modification consists in replacing two of the results used above by the second part of (a) and [12, Theorem 6.1(b)] or [9, Theorem 1(b)].

The next proposition will be used in establishing Theorem 1 in Sect. 5 .

Proposition 8 Suppose $\mu, \mu_{j} \in b a_{+}(\mathfrak{M})$ are such that $\sum_{j=1}^{\infty} \mu_{j}=\mu$ and $\mu_{j} \wedge \mu_{j^{\prime}}=$ 0 whenever $j \neq j^{\prime}$. Then the following two conditions are equivalent:

(i) $B_{E(\mu)}=B_{\text {extr } E(\mu)}$;

(ii) $B_{E\left(\mu_{j}\right)}=B_{\text {extr } E\left(\mu_{j}\right)}$ for each $j$.

Proof Suppose (i) holds, and fix $j$. In view of [14, pp. 202-203, (4) and (5)], we then get

$$
B_{E(\mu)} \subset B_{\mathrm{extr}} E\left(\mu_{j}\right)+B_{\mathrm{extr} E\left(\mu-\mu_{j}\right)} .
$$

It follows from Proposition 7(a) that

$$
E\left(\mu_{j}\right) \subset B_{\text {extr } E\left(\mu_{j}\right)}+B_{\text {extr } E\left(\mu-\mu_{j}\right)} .
$$

An application of [14, p. 203, (5)] now yields the nontrivial inclusion " $C$ " of (ii).

Suppose (ii) holds. Using Proposition 7(c), we then get

$$
\bigcup_{j=1}^{\infty} E\left(\mu_{j}\right) \subset B_{\bigcup_{j=1}^{\infty} \operatorname{extr} E\left(\mu_{j}\right)}=B_{\mathrm{extr}} E(\mu) .
$$


This together with Proposition 7(b) implies the nontrivial inclusion " $\subset$ " of (i).

The next result is a minor modification of [12, Proposition 7.1]. It will be used in establishing Lemmas 4 and 5.

Proposition 9 Let $\mu \in$ ult $(\mathfrak{M})$ and let $Z$ denote the Stone space of $\mathfrak{R} / \mathfrak{J}_{\mu}$. Then there exists a linear mapping $T: b a(\mathrm{CO}(Z)) \rightarrow$ ba $(\Re)$ with the following properties:

$1^{\circ} T$ is an isometry and a lattice homomorphism;

$2^{\circ} T(p a(\mathrm{CO}(Z)))=E(\mu)$;

$3^{\circ} T \mid p a(\mathrm{CO}(Z))$ is a homeomorphism with respect to the corresponding weak [weak ${ }^{*}$ topologies.

Consequently, $|\operatorname{extr} E(\mu)|=|Z|$.

The next two lemmas will be used in the proofs of Theorem 1 in Sect. 5 and Theorem 3 in Sect. 6, respectively.

Lemma 4 Let $\mu \in$ ult $(\mathfrak{M})$. Then the following two conditions are equivalent:

(i) $B_{E(\mu)}=B_{\operatorname{extr} E(\mu)}$.

(ii) $\mathfrak{R} / \mathfrak{J}_{\mu}$ is superatomic.

Proof In the notation of Proposition 9, we have

$$
T(b a(\operatorname{CO}(Z)))=\left\{\varphi \in b a(\Re): \varphi \mid \mathfrak{J}_{\mu}=0\right\} .
$$

This follows from $1^{\circ}$ and $2^{\circ}$ thereof or by an inspection of the proof [12, Proposition 7.1]. Thus, the range of $T$ is a band in $b a(\Re)$. Therefore, combining Proposition 9 and Remark 2, we see that condition (i) is equivalent to the following one:

$$
B_{p a(\mathrm{CO}(Z))}=B_{u l t(\mathrm{CO}(Z))}
$$

Therefore, the equivalence of (i) and (ii) follows from Proposition 4.

The implication (ii) $\Rightarrow$ (vi) of the following lemma strengthens [14, Lemma 1].

Lemma 5 Let $\mu \in$ ult $(\mathfrak{M})$. Then the following six conditions are equivalent:

(i) $E(\mu)$ is band dominated;

(ii) extr $E(\mu)$ is band dominated;

(iii) $E(\mu)$ is separable;

(iv) extr $E(\mu)$ is separable;

(v) $\mathfrak{R} / \mathfrak{J}_{\mu}$ is countable and superatomic;

(vi) extr $E(\mu)$ is countable.

The proof is analogous to that of Lemma 4. We only need to appeal to Proposition 5 instead of Proposition 4.

We note that the equivalent conditions of Lemma 5 do not imply that $E(\mu)$ is weakly compact, in general (see [14, Remark 1]), while this last condition implies (i) for arbitrary $\mu \in b a_{+}(\mathfrak{M})$, by [3, Theorem IV.9.12] and (PB). The converse still holds under an additional assumption. 
Corollary 1 Let $\mu \in$ ba+ $(\mathfrak{M})$ and let $\mathfrak{R}$ be a $\sigma$-algebra. If $E(\mu)$ is band dominated, then it is weakly compact. Consequently, $B_{E(\mu)}=B_{\operatorname{extr}} E(\mu)$.

In view of (PB), this follows from [17, Theorem $1.3,(2) \Rightarrow(1)]$, the weak* compactness of $E(\mu)$ (see [12, Proposition 4.4(a)] or [8, Proposition 1(a)]) and Proposition 3 above.

\section{Coincidence of $B_{E(\mu)}$ and $B_{\operatorname{extr}} E(\mu)$}

Theorem 1 below is related to two earlier results of the author, both concerned with the atomic case. Namely, Theorem 7.9 of [12] gives four other conditions equivalent to those of Theorem 1, while Theorem 8 of [14] gives a condition equivalent to the coincidence of $A_{E(\mu)}$ and $A_{\operatorname{extr} E(\mu)}$.

Theorem 1 Let $\mu \in b a_{+}(\mathfrak{M})$ be atomic. Then the following two condition are equivalent:

(i) $B_{E(\mu)}=B_{\text {extr } E(\mu)}$;

(ii) $\mathfrak{R} / \mathfrak{J}_{\nu}$ is superatomic for each $\nu \in \mathcal{U}_{\mu}$.

This is a direct consequence of Proposition 8 and Lemma 4.

Part (b) of the next result is related to [14, Proposition 5], which is concerned with the coincidence of $A_{E(\mu)}$ and $A_{\operatorname{extr} E(\mu)}$ for atomic $\mu \in b a_{+}(\mathfrak{M})$ under condition (*).

Theorem 2 Let $\mathfrak{N}$ be an algebra of subsets of $\Omega$ with $\mathfrak{R}=(\mathfrak{M} \cup \mathfrak{N})_{b}$ and let $\mu \in$ $b a_{+}(\mathfrak{M})$.

(a) If $\mathfrak{N}$ is superatomic, then $B_{E(\mu)}=B_{\operatorname{extr} E(\mu)}$;

(b) Suppose $\mu$ is atomic and nonzero, and

(*) $\quad M \cap N \neq \varnothing$ for all $M \in \mathfrak{M}$ with $\mu(M)>0$ and nonempty $N \in \mathfrak{N}$.

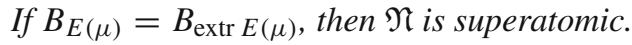

Proof (a): Fix $\varrho \in E(\mu)$. By assumption and [2, Theorem 5.3.6, (i) $\Rightarrow(\mathrm{v})], \varrho \mid \mathfrak{N}$ is atomic. In view of [11, Theorem 4(b)],

$$
\varrho \in \overline{\operatorname{conv}} \operatorname{extr} E(\mu) \text {, and so } \varrho \in B_{\operatorname{extr} E(\mu)} \text {. }
$$

This yields the nontrivial inclusion of (a).

(b): Choose $v \in \mathcal{U}_{\mu}$ with $v \neq 0$. By assumption and Theorem $1, \mathfrak{R} / \mathfrak{J}_{v}$ is superatomic. Consequently, so is $\mathfrak{N}$, by [14, Lemma 5].

Remark 4 We note that condition (*) or even the stronger condition that $M \cap N \neq \varnothing$ whenever $M \in \mathfrak{M}, N \in \mathfrak{N}$ and $M, N \neq \varnothing$ (cf. [15, p. 220]) does not imply the coincidence of $B_{E(\mu)}$ and $B_{\text {extr } E(\mu)}$, in general; see [12, Example 8.8] or [8, Example 4]. 


\section{Band domination and separability of $E(\mu)$ and $\operatorname{extr} E(\mu)$}

The next result is related to [14, Theorem 3], which is also concerned with the atomic case and gives two conditions equivalent to the ideal domination of extr $E(\mu)$. In this connection, we recall that condition (v) below can be restated as follows:

$(\mathrm{v})^{\prime} \operatorname{ult}\left(\mathfrak{R} / \mathfrak{J}_{v}\right)$ is countable for each $v \in \mathcal{U}_{\mu}$ (see Proposition 5, (v) $\Leftrightarrow$ (vi)).

Theorem 3 Let $\mu \in b a_{+}(\mathfrak{M})$ be atomic. Then the following five conditions are equivalent:

(i) $E(\mu)$ is band dominated;

(ii) extr $E(\mu)$ is band dominated;

(iii) $E(\mu)$ is separable;

(iv) extr $E(\mu)$ is separable;

(v) $\mathfrak{R} / \mathfrak{J}_{\nu}$ is countable and superatomic for each $v \in \mathcal{U}_{\mu}$.

Under these conditions, $B_{E(\mu)}=B_{\operatorname{extr}} E(\mu)$.

Proof By [12, Corollary 6.4], condition (iii) is equivalent to the following one:

$E(\nu)$ is separable for each $v \in \mathcal{U}_{\mu}$.

By the same result, condition (iv) is equivalent to the following one:

$\operatorname{extr} E(\nu)$ is separable for each $v \in \mathcal{U}_{\mu}$.

Therefore, Lemma 5 combined with Proposition 7 yields the equivalence of conditions (i)-(v). In view of Theorem 1, (v) implies the additional assertion.

The additional assertion of Theorem 3 does not imply its equivalence of conditions (i) $-(\mathrm{v})$, even for $\mu \in \operatorname{ult}(\mathfrak{M})$. This is seen by setting $\mathfrak{M}=\{\varnothing, \Omega\}$, where $\Omega$ is uncountable, and taking for $\Re$ an arbitrary uncountable superatomic algebra of subsets of $\Omega$ (cf. Proposition 4 and Remark 2).

A part of Theorem 3 holds, in fact, for arbitrary $\mu \in b a_{+}(\mathfrak{M})$, but a different, much less elementary argument is required.

Theorem $3^{\prime}$ For $\mu \in b a_{+}(\mathfrak{M})$ the following two conditions are equivalent:

(iii) $E(\mu)$ is separable;

(iv) extr $E(\mu)$ is separable.

Under these conditions, $B_{E(\mu)}=B_{\operatorname{extr} E(\mu)}$.

Proof Recall that, in view of [12, Proposition 4.4(a)] or [8, Proposition 1(a)], $E(\mu)$ is weak* compact. Suppose (iv) holds. According to a general result of Haydon [5, Theorem 3.3] and Kadec and Fonf [6, Theorem 1], we then have

$$
E(\mu)=\overline{\operatorname{conv}} \operatorname{extr} E(\mu) .
$$

This implies (iii) and the addition assertion. 
Also some other implications between conditions (i)-(v) of Theorem 3 hold for arbitrary $\mu \in b a_{+}(\mathfrak{M})$, either by Proposition $1(\mathrm{~b})$ or for obvious reasons. However, (i), and the more so (ii), does not imply (iv), and the more so (iii), in general. Indeed, if $\mathfrak{R}=(\mathfrak{M} \cup\{E\})_{b}$, where $E \subset \Omega$, then $E(\mu)$ is order bounded, by [8, Theorem 1(a)] or [13, Corollary 4]. On the other hand, extr $E(\mu)$ can be of arbitrary cardinality $\mathfrak{m}^{\aleph_{0}}$, where $\mathfrak{m}$ is a cardinality $\geqslant 1$ (see [12, Example 12.7] or [10, Example 3]). The author does not know whether (ii) implies (i) and $B_{E(\mu)}=B_{\operatorname{extr} E(\mu)}$ for arbitrary $\mu \in b a_{+}(\mathfrak{M})$ (cf. Remark 4).

The next result complements [14, Proposition 3].

Proposition 10 Let $\mu \in b a_{+}(\mathfrak{M})$ have finite range. Then the following three conditions are equivalent:

(i) extr $E(\mu)$ is band dominated;

(ii) extr $E(\mu)$ is ideal dominated;

(iii) extr $E(\mu)$ is countable.

Proof The assumption implies that $\mu$ is atomic and $\mathcal{U}_{\mu}$ is finite (see [2, Lemma 11.1.3] and [12, Lemma 3.2]). Suppose (i) holds. By Proposition 7(c) and Lemma 5, (ii) $\Rightarrow$ (vi), extr $E(v)$ is then countable for each $v \in \mathcal{U}_{\mu}$. Using [14, p. 202, formula (2)], we see that (iii) holds.

According to [14, Proposition 1], (iii) implies (ii). Clearly, (ii) implies (i).

Remark 5 The implication (i) $\Rightarrow$ (ii) of Proposition 10 does not hold for arbitrary atomic $\mu \in b a_{+}(\mathfrak{M})$; see [14, Example 2], and apply Theorem $3,(\mathrm{v}) \Rightarrow$ (ii).

Theorem 4 Let $\mathfrak{N}$ be an algebra of subsets of $\Omega$ with $\mathfrak{R}=(\mathfrak{M} \cup \mathfrak{N})_{b}$ and let $\mu \in$ $b a_{+}(\mathfrak{M})$.

(a) If $\mathfrak{N}$ is countable and superatomic and $\mu$ is atomic, then $E(\mu)$ is band dominated.

(b) Suppose

(*) $\quad M \cap N \neq \varnothing$ for all $M \in \mathfrak{M}$ with $\mu(M)>0$ and nonempty $N \in \mathfrak{N}$.

If $\operatorname{extr} E(\mu)$ is band dominated, then $\mathfrak{N}$ is countable and superatomic or $\mu=0$. Consequently,

$$
B_{E(\mu)}=B_{\text {extr } E(\mu)} .
$$

Proof (a): By [14, Lemma 5], $\mathfrak{R} / \mathfrak{J}_{\nu}$ is a homomorphic image of $\mathfrak{N}$ for each $v \in \mathcal{U}_{\mu}$ with $v \neq 0$. Thus, the assertion follows from Theorem $3,(v) \Rightarrow$ (i).

(b): Without loss of generality, we assume that $\mu(\Omega)=1$. Define the mapping

$$
R: b a(\mathfrak{R}) \rightarrow b a(\mathfrak{N}) \quad \text { by } \quad R(\varphi)=\varphi \mid \mathfrak{N} \text { for } \varphi \in b a(\mathfrak{R}) .
$$

We then have

$$
u l t(\mathfrak{N}) \subset R(\operatorname{extr} E(\mu))
$$

according to [12, Proposition 12.4] or [10, Proposition 2]. By assumption, extr $E(\mu)$ is contained in $B_{\tau}$ for some $\tau \in b a_{+}(\mathfrak{R})$. Therefore, ult $(\mathfrak{N})$ is contained 
in $B_{R(\tau)}$, by (PB). Proposition 5 , (ii) $\Rightarrow(\mathrm{v})$, now implies that $\mathfrak{N}$ is countable and superatomic. The final part of (b) is, therefore, a consequence of Theorem 2(a).

The author does not know whether Theorem 4(a) holds for arbitrary separable $\mu \in b a_{+}(\mathfrak{M})$ (cf. Remark 1). For $\mathfrak{N}$ generated by a countable family of pairwise disjoint subsets of $\Omega$ this is the case, even without the separability assumption (see [8, Theorem 1(b)] and (PB)).

The next result is a direct consequence of Theorem 4. It is worth-while to compare it with [13, Corollary 2] and [14, Theorem 7], which both also assume condition (*). They are concerned with order boundedness of $E(\mu)$ and ideal domination of extr $E(\mu)$, respectively.

Corollary 2 Let $\mu \in b a_{+}(\mathfrak{M})$ be atomic. In the setting of Theorem 4, with (*) assumed, the following two conditions are equivalent:

(i) $E(\mu)$ is band dominated;

(ii) $\mathfrak{N}$ is countable and superatomic or $\mu=0$.

Finally, we shall present a sufficient condition that $E(\mu)$ be separable.

Theorem 5 Let $\mu \in b a_{+}(\mathfrak{M})$ be separable and let $\mathfrak{R}=(\mathfrak{M} \cup \mathfrak{E})_{b}$, where $\mathfrak{E}$ is a countable family of pairwise disjoint subsets of $\Omega$. Then $E(\mu)$ is separable.

Proof By [8, Theorem 1(b)] and (PB), there exists $\tau \in E(\mu)$ such that $E(\mu) \subset B_{\tau}$. Clearly, $\tau$ satisfies the assumptions of Lemma 3 , and so is separable. Therefore, $E(\mu)$ is separable, by Lemma 2.

Needless to say, the countability assumption on $\mathfrak{E}$ is essential for the validity of Theorem 5 (cf. Proposition 5). This is also the case of the separability assumption on $\mu$, even if $\mathfrak{E}$ consists of only one set (see the passage following Theorem $3^{\prime}$ ). Also the disjointness assumption on $\mathfrak{E}$ is essential, as Example 3 of [8] combined with Proposition 1(b) and (PB) shows. Here is a simpler example to the same effect.

Example 2 (cf. Proposition 5). Let $\Omega$ be the Cantor set, and set $\mathfrak{M}=\{\varnothing, \Omega\}$ and $\Re=\operatorname{CO}(\Omega)$. Denote by $\delta_{\omega}$, where $\omega \in \Omega$, the Dirac quasi-measure on $\Re$ concentrated at $\omega$. Clearly, the set $\left\{\delta_{\omega}: \omega \in \Omega\right\}$ is (uniformly) discrete, and is contained in $E(\mu)$, where $\mu \in \operatorname{ult}(\mathfrak{M})$.

Postscript. More results on the separability of $E(\mu)$ will be presented in another paper by the author (in preparation).

Open Access This article is distributed under the terms of the Creative Commons Attribution 4.0 International License (http://creativecommons.org/licenses/by/4.0/), which permits unrestricted use, distribution, and reproduction in any medium, provided you give appropriate credit to the original author(s) and the source, provide a link to the Creative Commons license, and indicate if changes were made.

\section{References}

1. Aliprantis, C.D., Burkinshaw, O.: Positive Operators. Academic Press, Orlando (1985) 
2. Bhaskara Rao, K.P.S., Bhaskara Rao, M.: Theory of Charges. A Study of Finitely Additive Measures. Academic Press, London (1983)

3. Dunford, N., Schwartz, J.T.: Linear Operators. Part I. Interscience Publ., New York (1958)

4. Fremlin, D.H., Plebanek, G.: Large families of mutually singular Radon measures. Bull. Pol. Acad. Sci. Math. 51, 169-174 (2003)

5. Haydon, R.: An extreme point criterion for separability of a dual Banach space, and a new proof of a theorem of Corson. Quart. J. Math. Oxford 27(3), 379-385 (1976)

6. Kadec, M.I., Fonf, V.P.: Some properties of the set of extreme points of the unit ball of a Banach space. Mat. Zametki 20, 315-320 (1976) (Russian). Eng. transl.: Math. Notes 20, 737-739 (1976)

7. Koppelberg, S.: In: Monk, J.D. (ed.) Handbook of Boolean Algebras, vol. 1. North-Holland, Amsterdam (1989)

8. Lipecki, Z.: On compactness and extreme points of some sets of quasi-measures and measures. Manuscripta Math. 86, 349-365 (1995)

9. Lipecki, Z.: On compactness and extreme points of some sets of quasi-measures and measures. II. Manuscripta Math. 89, 395-406 (1996)

10. Lipecki, Z.: Cardinality of the set of extreme extensions of a quasi-measure. Manuscripta Math. 104, 333-341 (2001)

11. Lipecki, Z.: Cardinality of some convex sets and of their sets of extreme points. Colloq. Math. 123, 133-147 (2011)

12. Lipecki, Z.: Compactness and extreme points of the set of quasi-measure extensions of a quasi-measure. Dissertationes Math. (Rozprawy Mat.) 493, 59 pp. (2013)

13. Lipecki, Z:: Order boundedness and weak compactness of the set of quasi-measure extensions of a quasi-measure. Comment. Math. Univ. Carolin. 56, 331-345 (2015)

14. Lipecki, Z.: Order-theoretic properties of some sets of quasi-measures. Comment. Math. Univ. Carolin. 58, 197-212 (2017)

15. Marczewski, E.: Measures in almost independent fields. Fund. Math. 38, 217-229 (1951); reprinted in: Marczewski, E.: Collected Mathematical Papers. Institute of Mathematics, Polish Academy of Sciences, Warszawa, pp. 413-425 (1996)

16. Wnuk, W.: Banach Lattices with Order Continuous Norms. PWN_-Polish Sci. Publ., Warszawa (1999)

17. Zhang, X.-D.: On weak compactness in spaces of measures. J. Funct. Anal. 143, 1-9 (1997) 